\title{
A class of generalized pseudo-splines
}

\section{Zhitao Zhuang* and Jianwei Yang}

\section{${ }^{*}$ Correspondence:}

zhuangzhitao@ncwu.edu.cn

College of Mathematics and

Information Science, North China

University of Water Resources and

Electric Power, Zhengzhou, 450011,

P.R. China

\begin{abstract}
In this paper, a class of refinable functions is given by smoothening pseudo-splines in order to get divergence free and curl free wavelets. The regularity and stability of them are discussed. Based on that, the corresponding Riesz wavelets are constructed.
\end{abstract}

Keywords: pseudo-spline; wavelets; scaling function

\section{Introduction}

We denote by $\mathbb{Z}$ and $\mathbb{R}$ the set of integers and real numbers, respectively. Let $L_{p}(\mathbb{R})$ stand for the classical Lebesgue space

$$
L_{p}(\mathbb{R}):=\left\{f, \int_{\mathbb{R}}|f(t)|^{p} d t<+\infty\right\}
$$

with the norm $\|f\|_{p}=\left(\int_{\mathbb{R}}|f(t)|^{p} d t\right)^{\frac{1}{p}}$ and $L_{\infty}(\mathbb{R})$ consisting of all Lebesgue measurable and bounded functions on $\mathbb{R}$. Similarly, the discrete space $\ell_{p}(\mathbb{Z}):=\left\{\left\{a_{n}\right\}, \sum_{n}\left|a_{n}\right|^{p}<+\infty, n \in\right.$ $\mathbb{Z}\}$ with $\left\|\left\{a_{n}\right\}\right\|_{p}=\left(\sum_{n}\left|a_{n}\right|^{p}\right)^{\frac{1}{p}}$. As usual, given $f \in L_{1}(\mathbb{R}) \cap L_{2}(\mathbb{R})$, its Fourier transform is defined by

$$
\hat{f}(\omega):=\int_{\mathbb{R}} f(x) e^{-i x \omega} d x
$$

on $\mathbb{R}$. The Fourier transform of a function in $L_{2}(\mathbb{R})$ is understood as the unitary extension. We write $h=f * g$ for the convolution $h(x)=\int_{\mathbb{R}} f(x-t) g(t) d t$, defined for any pair of functions $f$ and $g$ such that the integral exists almost everywhere. Clearly, $\hat{h}(\omega)=\hat{f}(\omega) \hat{g}(\omega)$ in the frequency domain, when all the Fourier transforms exist in that formula. Given $g \in L_{2}(\mathbb{R}),\{g(x-k), k \in \mathbb{Z}\}$ is called a Riesz basis of its linearly generating space, if for each $\left\{\lambda_{k}\right\} \in \ell_{2}(\mathbb{Z})$ there exist two positive constants $A$ and $B$ such that

$$
A \sum_{k \in \mathbb{Z}} \lambda_{k}^{2} \leq\left\|\sum_{k \in \mathbb{Z}} \lambda_{k} g(x-k)\right\|_{2}^{2} \leq B \sum_{k \in \mathbb{Z}} \lambda_{k}^{2}
$$

The numbers $A, B$ are called lower Riesz bound and upper Riesz bound, respectively.

Multiresolution analysis provides a classical method to construct wavelets.

Definition 1 A multiresolution analysis of $L_{2}(\mathbb{R})$ means a sequence of closed linear subspaces $V_{j}$ of $L_{2}(\mathbb{R})$ which satisfies

(c) 2014 Zhuang and Yang; licensee Springer. This is an Open Access article distributed under the terms of the Creative Commons Attribution License (http://creativecommons.org/licenses/by/2.0), which permits unrestricted use, distribution, and reproduction in any medium, provided the original work is properly cited. 
(i) $V_{j} \subset V_{j+1}, j \in \mathbb{Z}$,

(ii) $f(x) \in V_{j}$ if and only if $f(2 x) \in V_{j+1}$,

(iii) $\overline{\bigcup_{j \in \mathbb{Z}} V_{j}}=L_{2}(\mathbb{R})$ and $\bigcap_{j \in \mathbb{Z}} V_{j}=\{0\}$,

(iv) there exists a function $\phi \in L_{2}(\mathbb{R})$ such that $\{\phi(x-k), k \in \mathbb{Z}\}$ forms a Riesz basis of $V_{0}$.

The function $\phi$ in Definition 1 is said to be a scaling function, if it satisfies

$$
\phi(x)=\sum_{k} a_{k} \phi(2 x-k)
$$

for some sequence $\left\{a_{k}\right\} \in \ell_{2}(\mathbb{Z})$. Define the Fourier series $\hat{c}$ of a sequence $\left\{c_{k}\right\} \in \ell_{2}(\mathbb{Z})$ by

$$
\hat{c}(\xi):=\sum_{k \in \mathbb{Z}} c_{k} e^{-i k \xi}, \quad \xi \in \mathbb{R} .
$$

Then the refinement equation (1.2) becomes

$$
\hat{\phi}(\xi)=\hat{a}(\xi / 2) \hat{\phi}(\xi / 2), \quad \xi \in \mathbb{R} .
$$

The function $\hat{a}$ is called the refinement mask of $\phi$. The pseudo-spline of Type I was first introduced in [1] to construct tight framelets. The pseudo-spline of Type II was first studied by Dong and Shen in [2]. There have been many developments in the theory of pseudosplines over the past ten years [3, 4]. Its applications in image denoising and image inpainting are also very extensive $[5,6]$. The pseudo-spline is defined by its refinement mask. The refinement mask of a pseudo-spline of Type I with order $(m, \ell)$ is given by

$$
\left.{ }_{1} \hat{a}(\xi)\right|^{2}:=\left|{ }_{1} \hat{a}_{m, \ell}(\xi)\right|^{2}:=\cos ^{2 m}(\xi / 2) \sum_{j=0}^{\ell}\left(\begin{array}{c}
m+\ell \\
j
\end{array}\right) \sin ^{2 j}(\xi / 2) \cos ^{2(\ell-j)}(\xi / 2)
$$

and the refinement of a pseudo-spline of Type II with order $(m, \ell)$ is given by

$$
{ }_{2} \hat{a}(\xi):={ }_{2} \hat{a}_{m, \ell}(\xi):=\cos ^{2 m}(\xi / 2) \sum_{j=0}^{\ell}\left(\begin{array}{c}
m+\ell \\
j
\end{array}\right) \sin ^{2 j}(\xi / 2) \cos ^{2(\ell-j)}(\xi / 2) .
$$

The mask of Type I is obtained by taking the square root of the mask of Type II using the Fejér-Riesz lemma [7], i.e. ${ }_{2} \hat{a}(\xi)=\left.{ }_{1} \hat{a}(\xi)\right|^{2}$. The corresponding pseudo-spline can be defined in terms of their Fourier transform, i.e.

$$
{ }_{k} \hat{\phi}_{m, \ell}(\xi):=\prod_{j=1}^{\infty} \hat{k}_{m, \ell}\left(2^{-j} \xi\right), \quad k=1,2
$$

In order to smoothen the pseudo-spline, one can use the convolution method. Take the smoothed pseudo-spline

$$
\phi_{n, m, \ell}=\phi_{m, \ell} * \underbrace{\chi_{\left[-\frac{1}{2}, \frac{1}{2}\right]} * \cdots * \chi_{\left[-\frac{1}{2}, \frac{1}{2}\right]}}_{n-m},
$$


where $\chi_{\left[-\frac{1}{2}, \frac{1}{2}\right]}$ denotes the characteristic function of interval $\left[-\frac{1}{2}, \frac{1}{2}\right]$ and $n \geq m$. This is equivalent to

$$
\hat{\phi}_{n, m, \ell}(\xi)=\hat{\phi}_{m, \ell}(\xi)\left(\frac{\sin (\xi / 2)}{\xi / 2}\right)^{n-m}
$$

Thus the symbol of $\phi_{n, m, \ell}$ becomes

$$
\hat{a}_{n, m, \ell}(\xi)=\hat{\phi}_{n, m, \ell}(2 \xi) / \hat{\phi}_{n, m, \ell}(\xi)=\hat{a}_{m, \ell}(\xi)(\cos (\xi / 2))^{n-m}
$$

Therefore, we define the smoothed pseudo-spline by its refinement mask for Type I:

$$
\left|{ }_{1} \hat{a}_{n, m, \ell}\right|^{2}:=\cos ^{2 n}(\xi / 2) \sum_{j=0}^{\ell}\left(\begin{array}{c}
m+\ell \\
j
\end{array}\right) \sin ^{2 j}(\xi / 2) \cos ^{2(\ell-j)}(\xi / 2)
$$

and for Type II:

$$
{ }_{2} \hat{\phi}_{r, m, \ell}:=\cos ^{r}(\xi / 2) \sum_{j=0}^{\ell}\left(\begin{array}{c}
m+\ell \\
j
\end{array}\right) \sin ^{2 j}(\xi / 2) \cos ^{2(\ell-j)}(\xi / 2)
$$

where $r \geq 2 m$. When $r=2 m$, it is the pseudo-spline. When $r \neq 2 m$, it can be considered as an extension of pseudo-spline. Define the translated form of the Type II by

$$
{ }_{T} \hat{\phi}_{r, m, \ell}(\xi):=e^{-i r \frac{\xi}{2}}{ }_{2} \hat{\phi}_{r, m, \ell}(\xi)
$$

Then we get the differential relation

$$
{ }_{T} \phi_{r+1, m, \ell}^{\prime}(x)={ }_{T} \phi_{r, m, \ell}(x)-{ }_{T} \phi_{r, m, \ell}(x-1) .
$$

This inherits the property of a B-spline.

Remark 1 One may think that smoothing the pseudo-splines by convolving them with B-splines seems unnecessary since one can simply increase $m$ of the original pseudosplines. However, by increasing $m$, we cannot get the differential relation (1.6), which is important for the construction of divergence free wavelets and curl free wavelets in the analysis of incompressible turbulent flows $[8,9]$.

Remark 2 Similar to the definition of (1.5), we can define a smoothed dual pseudo-spline by its refinement mask,

$$
\hat{b}_{n, m, \ell}(\xi)=e^{i \xi / 2} \cos ^{2 n+1}(\xi / 2) \sum_{j=0}^{\ell}\left(\begin{array}{c}
m+1 / 2+\ell \\
j
\end{array}\right) \sin ^{2 j}(\xi / 2) \cos ^{2(\ell-j)}(\xi / 2)
$$

as an extension of dual pseudo-splines in [3] and get the corresponding wavelets.

Remark 3 In addition, one can assume $n \in \mathbb{R}$ in (1.5) and (1.7), as a generalization of fractional splines in [10]. 


\section{Some lemmas}

This section gives some lemmas that will be used to prove several results of this paper. We start with some results from [2].

Lemma 1 [2] For given nonnegative integers $m, j, \ell$,

$$
2^{\ell}\left(\begin{array}{c}
m+\ell \\
\ell
\end{array}\right)^{\frac{1}{2}} \leq \sum_{j=0}^{\ell}\left(\begin{array}{c}
m+\ell \\
j
\end{array}\right) \text { for all } m \geq 1 \text { and } 0 \leq \ell \leq m-1 \text {. }
$$

This lemma will be used in Section 4 in order to prove the Riesz basis property of wavelets. Define $P_{m, \ell}(y):=\sum_{j=0}^{\ell}\left(\begin{array}{c}m+\ell \\ j\end{array}\right) y^{j}(1-y)^{\ell-j}, R_{m, \ell}(y):=(1-y)^{m} P_{m, \ell}(y)$ and $R_{r, m, \ell}=$ $(1-y)^{\frac{r}{2}} P_{m, \ell}(y)$ where $y=\sin ^{2}(\xi / 2), r, m, \ell$ are nonnegative integers and $r \geq 2 m$. Then one can find that

$$
R_{m, \ell}\left(\sin ^{2}(\xi / 2)\right)={ }_{2} \hat{a}_{m, \ell}(\xi) \text { and } R_{r, m, \ell}\left(\sin ^{2}(\xi / 2)\right)={ }_{2} \hat{a}_{r, m, \ell}(\xi)
$$

and the following lemma holds.

Lemma 2 [2] For nonnegative integers $m$ and $\ell$ with $\ell \leq m-1$, let $P_{m, \ell}$ and $R_{m, \ell}$ be the polynomials defined above. Then

(1) $P_{m, \ell}(y)=\sum_{j=0}^{\ell}\left(\begin{array}{c}m-1+j \\ j\end{array}\right) y^{j}$;

(2) $R_{m, \ell}^{\prime}(y)=-(m+\ell)\left(\begin{array}{c}m+\ell-1 \\ \ell\end{array}\right) y^{\ell}(1-y)^{m-1}$.

With the two lemmas in hand, the basic property of the polynomial $R_{r, m, \ell}$, which will be used in Section 4, is given.

Lemma 3 For nonnegative integers $r, m$ and $\ell$,

(1) define $Q(y):=R_{r, m, \ell}(y)+R_{r, m, \ell}(1-y)$; then

$$
\min _{y \in[0,1]} Q(y)=Q\left(\frac{1}{2}\right)=2^{1-\frac{r}{2}-\ell} \sum_{j=0}^{\ell}\left(\begin{array}{c}
m+\ell \\
j
\end{array}\right)
$$

(2) define $S(y):=R_{r, m, \ell}^{2}(y)+R_{r, m, \ell}^{2}(1-y)$; then

$$
\min _{y \in[0,1]} S(y)=S\left(\frac{1}{2}\right)=2^{1-r-2 \ell}\left(\sum_{j=0}^{\ell}\left(\begin{array}{c}
m+\ell \\
j
\end{array}\right)\right)^{2} .
$$

Proof Since $R_{r, m, \ell}(y)=(1-y)^{\frac{r}{2}-m} R_{m, \ell}(y)$, its derivative is

$$
R_{r, m, \ell}^{\prime}(y)=-\left(\frac{r}{2}-m\right)(1-y)^{\frac{r}{2}-m-1} R_{m, \ell}(y)+(1-y)^{\frac{r}{2}-m} R_{m, \ell}^{\prime}(y) .
$$

So the derivative is $Q^{\prime}(y)=R_{r, m, \ell}^{\prime}(y)+R_{r, m, \ell}^{\prime}(1-y)=I+I I$, where

$$
I=\left(\frac{r}{2}-m\right) y^{\frac{r}{2}-m-1} R_{m, \ell}(1-y)-\left(\frac{r}{2}-m\right)(1-y)^{\frac{r}{2}-m-1} R_{m, \ell}(y)
$$


and

$$
I I=(1-y)^{\frac{r}{2}-m} R_{m, \ell}^{\prime}(y)-y^{\frac{r}{2}-m} R_{m, \ell}^{\prime}(1-y) .
$$

Now, we compute them, respectively. For $I$, by using (1) of Lemma 2, one has

$$
I=\left(\frac{r}{2}-m\right) \sum_{j=0}^{\ell}\left(\begin{array}{c}
m-1+j \\
j
\end{array}\right)\left[y^{\frac{r}{2}-1}(1-y)^{j}-y^{j}(1-y)^{\frac{r}{2}-1}\right] .
$$

For $I I$, by using (2) of Lemma 2, one has

$$
I I=(m+\ell)\left(\begin{array}{c}
m+\ell-1 \\
\ell
\end{array}\right)\left[y^{\frac{r}{2}-1}(1-y)^{\ell}-(1-y)^{\frac{r}{2}-1} y^{\ell}\right] .
$$

For $j=0, \ldots, \ell$, since $y^{\frac{r}{2}-1}(1-y)^{j} \leq(1-y)^{\frac{r}{2}-1} y^{j}$ for all $y \in\left[0, \frac{1}{2}\right]$ and $y^{\frac{r}{2}-1}(1-y)^{j} \geq(1-y)^{\frac{r}{2}-1} y^{j}$ for all $y \in\left[\frac{1}{2}, 1\right]$, one has

$$
Q^{\prime}(y)=I+I I \begin{cases}\leq 0, & y \in\left[0, \frac{1}{2}\right] \\ \geq 0, & y \in\left[\frac{1}{2}, 1\right]\end{cases}
$$

This means $Q(y)$ reaches its minimum value at the point $y=1 / 2$. Furthermore,

$$
Q(1 / 2)=2 R_{r, m, \ell}(1 / 2)=2^{1-\frac{r}{2}} P_{m, \ell}(1 / 2)=2^{1-\frac{r}{2}-\ell} \sum_{j=0}^{\ell}\left(\begin{array}{c}
m+\ell \\
j
\end{array}\right) .
$$

This completes the proof of (1). For (2) of this lemma, since

$$
S(y)=R_{r, m, \ell}^{2}(y)+R_{r, m, \ell}^{2}(1-y)=(1-y)^{r-2 m} R_{m, \ell}^{2}(y)+y^{r-2 m} R_{m, \ell}^{2}(1-y),
$$

we have $S^{\prime}(y)=I I I+I V$, where

$$
I I I=(r-2 m)\left[y^{r-2 m-1} R_{m, \ell}^{2}(1-y)-(1-y)^{r-2 m-1} R_{m, \ell}^{2}(y)\right]
$$

and

$$
I V=2(1-y)^{r-2 m} R_{m, \ell}(y) R_{m, \ell}^{\prime}(y)-2 y^{r-2 m} R_{m, \ell}(1-y) R_{m, \ell}^{\prime}(1-y) .
$$

For $I I I$, by (1) of Lemma 2, we have

$$
\begin{aligned}
I I I= & (r-2 m)\left[y^{r-1} P_{m, \ell}^{2}(1-y)-(1-y)^{r-1} P_{m, \ell}^{2}(y)\right] \\
= & (r-2 m)\left(\left(\sum_{j=0}^{\ell}\left(\begin{array}{c}
m-1+j \\
j
\end{array}\right) y^{\frac{1}{2}(r-1)}(1-y)^{j}\right)^{2}\right. \\
& \left.-\left(\sum_{j=0}^{\ell}\left(\begin{array}{c}
m-1+j \\
j
\end{array}\right)(1-y)^{\frac{1}{2}(r-1)} y^{j}\right)^{2}\right)
\end{aligned}
$$




$$
\begin{aligned}
= & (r-2 m)\left(\sum_{j=0}^{\ell}\left(\begin{array}{c}
m-1+j \\
j
\end{array}\right)\left(y^{\frac{1}{2}(r-1)}(1-y)^{j}+(1-y)^{\frac{1}{2}(r-1)} y^{j}\right)\right) \\
& \times\left(\sum_{j=0}^{\ell}\left(\begin{array}{c}
m-1+j \\
j
\end{array}\right)\left(y^{\frac{1}{2}(r-1)}(1-y)^{j}-(1-y)^{\frac{1}{2}(r-1)} y^{j}\right)\right) .
\end{aligned}
$$

For $I V$, by (2) of Lemma 2, we have

$$
\begin{aligned}
\frac{I V}{2} & =(1-y)^{r-2 m} P_{m, \ell}(y) R_{m, \ell}^{\prime}(y)-y^{r-2 m} P_{m, \ell}(1-y) R_{m, \ell}^{\prime}(1-y) \\
& =R_{m, \ell}^{\prime}(y) \sum_{j=0}^{\ell}\left(\begin{array}{c}
m-1+j \\
j
\end{array}\right) y^{j}(1-y)^{r-2 m}-R_{m, \ell}^{\prime}(1-y) \sum_{j=0}^{\ell}\left(\begin{array}{c}
m-1+j \\
j
\end{array}\right)(1-y)^{j} y^{r-2 m} \\
& =(m+\ell)\left(\begin{array}{c}
m-1+j \\
j
\end{array}\right)\left(\sum_{j=0}^{\ell}\left(\begin{array}{c}
m-1+j \\
j
\end{array}\right)\left(y^{r-1}(1-y)^{\ell+j}-y^{\ell+j}(1-y)^{r-1}\right)\right) .
\end{aligned}
$$

Since

$$
y^{\frac{1}{2}(r-1)}(1-y)^{j}-(1-y)^{\frac{1}{2}(r-1)} y^{j} \begin{cases}\leq 0, & y \in\left[0, \frac{1}{2}\right] \\ \geq 0, & y \in\left[\frac{1}{2}, 1\right]\end{cases}
$$

and for every $j$,

$$
y^{r-1}(1-y)^{\ell+j}-y^{\ell+j}(1-y)^{r-1} \begin{cases}\leq 0, & y \in\left[0, \frac{1}{2}\right] \\ \geq 0, & y \in\left[\frac{1}{2}, 1\right]\end{cases}
$$

we have

$$
S^{\prime}(y)=I I I+I V \begin{cases}\leq 0, & y \in\left[0, \frac{1}{2}\right] \\ \geq 0, & y \in\left[\frac{1}{2}, 1\right]\end{cases}
$$

This means $S(y)$ reaches its minimum at point $y=1 / 2$. Furthermore, we have

$$
S(1 / 2)=2\left(2^{-\frac{r}{2}} P_{m, \ell}(y)\right)^{2}=2^{1-r-2 \ell}\left(\sum_{j=0}^{\ell}\left(\begin{array}{c}
m-1+j \\
j
\end{array}\right)\right)^{2} .
$$

This completes the lemma.

\section{Regularity and stability of scaling function}

In this section, we discuss the regularity and stability of a scaling function generated by the refinement mask of a smoothed pseudo-spline. Let

$$
\hat{\phi}(\xi):=\prod_{j=1}^{\infty} \hat{a}\left(2^{-j} \xi\right)
$$

Then the decay of $|\hat{\phi}|$ can be characterized by $|\hat{a}|$ as stated in the following theorem. 
Theorem 1 [2] Let $\hat{a}(\xi)$ be a refinement mask of the refinable function $\phi$ of the form

$$
|\hat{a}(\xi)|=\cos ^{n}(\xi / 2)|\mathcal{L}(\xi)|, \quad \xi \in[-\pi, \pi] .
$$

Suppose that

$$
\begin{aligned}
& |\mathcal{L}(\xi)| \leq\left|\mathcal{L}\left(\frac{2 \pi}{3}\right)\right| \text { for }|\xi| \leq \frac{2 \pi}{3} \\
& |\mathcal{L}(\xi) \mathcal{L}(2 \xi)| \leq\left|\mathcal{L}\left(\frac{2 \pi}{3}\right)\right|^{2} \text { for } \frac{2 \pi}{3} \leq|\xi| \leq \pi
\end{aligned}
$$

Then $|\hat{\phi}(\xi)| \leq C(1+|\xi|)^{-n+\kappa}$, with $\kappa=\log \left(\mathcal{L}\left(\mid \frac{2 \pi}{3}\right) \mid\right) / \log 2$, and this decay is optimal.

In order to use this lemma, one needs to consider the polynomial corresponding to $\mathcal{L}(\xi)$. In fact, Dong and Shen give an important proposition to estimate it in the following proposition.

Proposition 1 [2] Let $P_{m, \ell}(y)$ be defined as in Section 2, where $m, \ell$ are nonnegative integers with $\ell \leq m-1$. Then

$$
\begin{aligned}
& P_{m, \ell}(y) \leq P_{m, \ell}\left(\frac{3}{4}\right) \quad \text { for } y \in\left[0, \frac{3}{4}\right], \\
& P_{m, \ell}(y) P_{m, \ell}(4 y(1-y)) \leq\left(P_{m, \ell}\left(\frac{3}{4}\right)\right)^{2} \text { for } y \in\left[\frac{3}{4}, 1\right] .
\end{aligned}
$$

Combing Theorem 1 and Proposition 1, we have the following theorem, which characterizes the regularity of a smoothed pseudo-spline.

Theorem 2 Let ${ }_{2} \phi$ be the smoothed pseudo-spline of Type II with order $r, m, \ell$, then

$$
\left|{ }_{2} \hat{\phi}(\xi)\right| \leq C(1+|\xi|)^{-r+\kappa},
$$

where $\kappa=\log \left(P_{m, \ell}\left(\frac{3}{4}\right)\right) / \log 2$. Consequently, ${ }_{2} \phi \in C^{\alpha_{2}-\epsilon}$ where $\alpha_{2}=r-\kappa-1$. Furthermore, let ${ }_{1} \phi$ be the smoothed pseudo-spline of Type I with order $n, m, \ell$. Then

$$
\left|{ }_{1} \hat{\phi}(\xi)\right| \leq C(1+|\xi|)^{-n+\frac{\kappa}{2}} .
$$

Consequently, ${ }_{1} \phi \in C^{\alpha_{1}-\epsilon}$ with $\alpha_{1}=n-\frac{\kappa}{2}-1$.

Proof Notice that $|\mathcal{L}(\xi)|$ in Theorem 1 is exactly $P_{m, \ell}\left(\sin ^{2}\left(\frac{\xi}{2}\right)\right)$ and $4 y(1-y)=\sin ^{2}(\xi)$; one can easily prove this theorem by Theorem 1 and Proposition 1 .

This theorem shows ${ }_{k} \phi \in L_{2}(\mathbb{R})$ for $k=1$, 2. Since $r \geq 2 m$, the regularity of $\phi$ is better than a pseudo-spline but the support is longer. For $r=6, m=2, \ell=1$ the smoothed pseudo-spline ${ }_{2} \phi_{r, m, \ell}$ is shown in Figure 1.

Now, we consider the stability of the smoothed pseudo-spline. When $\phi$ is compactly supported in $L_{2}(\mathbb{R})$, it was shown by Jia and Micchelli [11] that the upper Riesz bound in 
Figure 1 The scaling function ${ }_{2} \phi_{3,2,1}$.

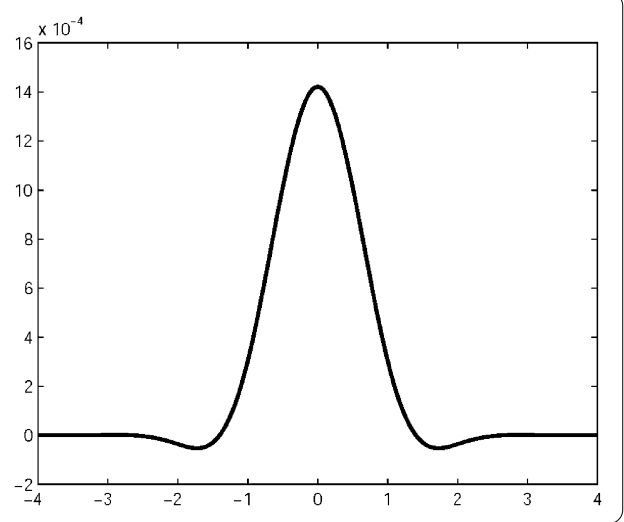

(1.1) always exists. Furthermore, they assert that the existence of a lower Riesz bound is equivalent to

$$
(\hat{\phi}(\xi+2 k \pi))_{k \in \mathbb{Z}} \neq \mathbf{0} \text { for all } \xi \in \mathbb{R}
$$

where $\mathbf{0}$ denotes the zero sequence in $\ell_{2}(\mathbb{Z})$. Since a smoothed pseudo-spline is compactly supported and belongs to $L_{2}(\mathbb{R})$ for $k=1,2$, the stability is equivalent to (3.1).

Theorem 3 Smoothed pseudo-splines are stable.

Proof By the definition of refinement mask, for each fixed $\frac{r}{2} \geq m \geq 1$ and for any $0 \leq \ell \leq$ $m-1, \cos ^{2 m}(\xi / 2) \leq{ }_{2} \hat{a}_{r, m, \ell}(\xi)$ holds for all $\xi \in \mathbb{R}$. Therefore, we have

$$
\left|\hat{B}_{r}(\xi)\right| \leq\left|\hat{\phi}_{r, m, \ell}(\xi)\right|
$$

where $B_{r}$ stands for the B-spline with order $r$. Since $B_{r}$ is stable, the vector $\left(\hat{B}_{r}(\xi+\right.$ $2 k \pi))_{k \in \mathbb{Z}} \neq \mathbf{0}$. Hence $\left(\hat{\phi}_{r, m, \ell}(\xi+2 k \pi)\right)_{k \in \mathbb{Z}} \neq \mathbf{0}$.

For a smoothed pseudo-spline of Type I, since ${ }_{2} \hat{a}_{2 n, m, \ell}(\xi)=\left.{ }_{1} \hat{a}_{n, m, \ell}\right|^{2}={ }_{1} \hat{a}_{n, m, \ell}(\xi)$. ${ }_{1} \hat{a}_{n, m, \ell}(-\xi)$, one has

$$
{ }_{2} \hat{\phi}_{2 n, m, \ell}(\xi)={ }_{1} \hat{\phi}_{n, m, \ell}(\xi) \cdot{ }_{1} \hat{\phi}_{n, m, \ell}(-\xi)
$$

Therefore, the set of zeros of ${ }_{1} \hat{\phi}_{n, m, \ell}(\xi)$ is contained in that of ${ }_{2} \hat{\phi}_{2 n, m, \ell}(\xi)$ and this guarantees the stability of ${ }_{1} \phi(\xi)$.

This theorem shows the stability of a smoothed pseudo-spline. From the definition of a Riesz basis, one can find that the translate of a smoothed pseudo-spline is also linearly independent.

\section{Riesz wavelets}

Since all smoothed pseudo-splines are compactly supported, refinable, stable in $L_{2}(\mathbb{R})$, the sequence of spaces $\left(V_{n}\right)_{n \in \mathbb{Z}}$ defined via Definition 1 forms an MRA. The corresponding wavelets can be constructed by the classical method. Define

$$
\hat{\psi}(\xi)=\hat{b}\left(\frac{\xi}{2}\right) \hat{\phi}\left(\frac{\xi}{2}\right), \quad \text { where } \hat{b}(\xi)=e^{-i \xi} \overline{\hat{a}(\xi+\pi)}
$$


and $X(\psi):=\left\{\psi_{n, k}=2^{n / 2} \psi\left(2^{n}-k\right), n, k \in \mathbb{Z}\right\}$. Then $X(\psi)$ is a Riesz basis. To prove this, the following theorem is needed.

Theorem 4 [2] Let $\hat{a}(\xi)$ be a finitely supported refinement mask of a refinable function $\phi \in L_{2}(\mathbb{R})$ with $\hat{a}(0)=1$ and $\hat{a}(\pi)=0$, such that $\hat{a}$ can be factorized into the form

$$
|\hat{a}(\xi)|=\cos ^{n}(\xi / 2)|\mathcal{L}(\xi)|, \quad \xi \in[-\pi, \pi],
$$

where $\mathcal{L}$ is the Fourier series of a finitely supported sequence with $\mathcal{L}(\pi) \neq 0$. Suppose that

$$
|\hat{a}(\xi)|^{2}+|\hat{a}(\xi+\pi)|^{2} \neq 0, \quad \xi \in[-\pi, \pi] .
$$

Define $\hat{\psi}(2 \xi):=e^{-i \xi} \overline{\hat{a}(\xi+\pi)} \hat{\phi}(\xi)$ and $\tilde{\mathcal{L}}:=\frac{\mathcal{L}(\xi)}{|\hat{a}(\xi)|^{2}+|\hat{a}(\xi+\pi)|^{2}}$. Assume that $\|\mathcal{L}(\xi)\|_{L_{\infty}(\mathbb{R})}<2^{n-1}$ and $\|\tilde{\mathcal{L}}(\xi)\|_{L_{\infty}(\mathbb{R})}<2^{n-1}$. Then $X(\psi)$ is a Riesz basis for $L_{2}(\mathbb{R})$.

From the above theorem, the key step is to estimate the upper Riesz bound of $|\mathcal{L}(\xi)|$ and $|\tilde{\mathcal{L}}(\xi)|$. Notice that

$$
\left|{ }_{1} \hat{a}_{n, m, \ell}(\xi)\right|^{2}={ }_{2} \hat{a}_{2 n, m, \ell}(\xi)=\cos ^{2 n}(\xi / 2) P_{m, \ell}\left(\sin ^{2}(\xi / 2)\right) .
$$

One has ${ }_{1} \mathcal{L}(\xi)\left|=\left(P_{m, \ell}\left(\sin ^{2}(\xi / 2)\right)\right)^{\frac{1}{2}},\right| \mathcal{L}_{2}(\xi) \mid=P_{m, \ell}\left(\sin ^{2}(\xi / 2)\right)$ and

$$
\left|\tilde{\mathcal{L}}_{1}\right|=\frac{\left(P_{m, \ell}(y)\right)^{\frac{1}{2}}}{R_{2 n, m, \ell}(y)+R_{2 n, m, \ell}(1-y)}, \quad\left|\tilde{\mathcal{L}}_{2}\right|=\frac{P_{m, \ell}(y)}{R_{r, m, \ell}^{2}(y)+R_{r, m, \ell}^{2}(1-y)} .
$$

Thus, we have the following theorem.

Theorem 5 Let ${ }_{k} \phi, k=1,2$ be the smoothed pseudo-spline of Types I and II with order $(r, n, m, \ell)$. The refinement masks ${ }_{k} a$ are given in (1.3) and (1.4). Define

$$
{ }_{k} \hat{\psi}(2 \xi):=e^{-i \xi} \overline{{ }_{k} \hat{a}(\xi+\pi)}{ }_{k} \hat{\phi}(\xi) .
$$

Then $X(\psi)$ forms a Riesz basis for $L_{2}(\mathbb{R})$.

Proof By (1) of Lemma 3, one obtains

$$
\begin{aligned}
\left\|_{1} \tilde{\mathcal{L}}\right\|_{L_{\infty}(\mathbb{R})} & =\sup _{y \in[0,1]} \frac{\left(P_{m, \ell}(y)\right)^{\frac{1}{2}}}{R_{2 n, m, \ell}(y)+R_{2 n, m, \ell}(1-y)} \\
& \leq \frac{\left(\begin{array}{c}
m+\ell \\
\ell
\end{array}\right)^{\frac{1}{2}}}{\min _{y \in[0,1]}\left(R_{2 n, m, \ell}(y)+R_{2 n, m, \ell}(1-y)\right)} \\
& \leq \frac{2^{n+\ell-1}\left(\begin{array}{c}
m+\ell \\
\ell
\end{array}\right)}{\sum_{j=0}^{\ell}\left(\begin{array}{c}
\frac{1}{2}+\ell \\
j
\end{array}\right)} .
\end{aligned}
$$

Applying Lemma 1 , one obtains $\left\|_{1} \tilde{\mathcal{L}}_{\infty}\right\| \leq 2^{n-1}<2^{n-\frac{1}{2}}$. Similarly, one can get

$$
\left\|_{2} \tilde{\mathcal{L}}\right\|_{L_{\infty}(\mathbb{R})} \leq 2^{r-1}<2^{r-\frac{1}{2}} .
$$




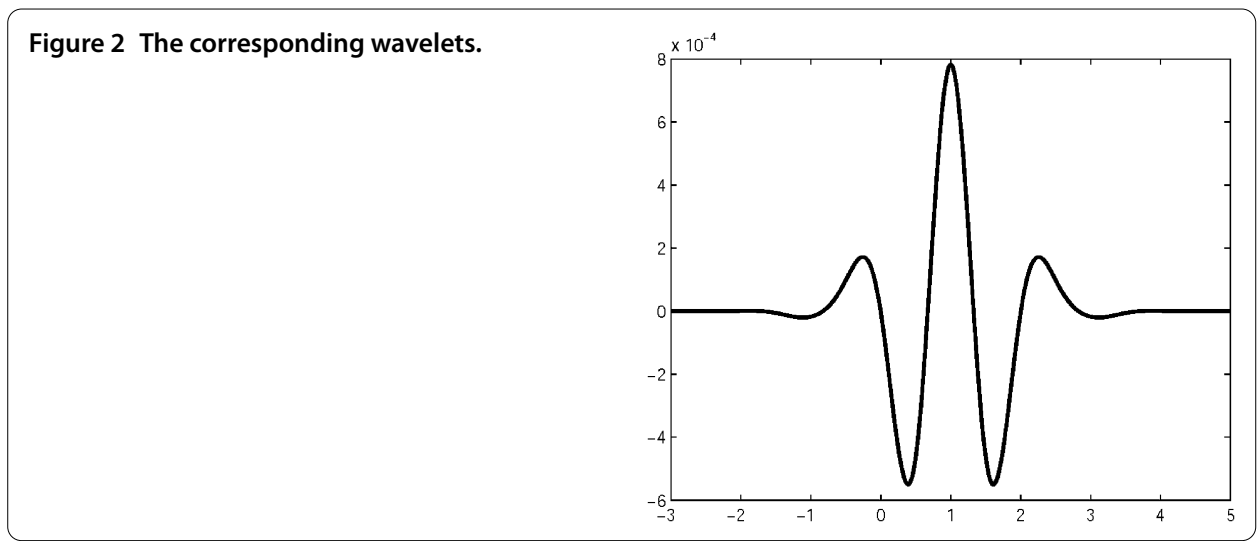

Notice that

$$
\left|{ }_{k} \hat{a}(\xi)\right|^{2}+\left|{ }_{k} \hat{a}(\xi+\pi)\right|^{2} \leq 1 \quad \text { for all } \xi \in \mathbb{R}
$$

Hence, ${ }_{k} \mathcal{L}(\xi)\left|\leq{ }_{k} \tilde{\mathcal{L}}(\xi)\right|$ for $k=1,2$. By using Theorem 4 , one gets the desired result.

By definition, the wavelets are also in $L_{2}(\mathbb{R})$ and have the same regularity as the scaling function. Still, the support is longer than for pseudo-spline wavelets. For $r=6, m=2, \ell=1$, the smoothed pseudo-spline ${ }_{2} \psi_{6,2,1}$ is shown in Figure 2.

\section{Competing interests}

The authors declare that they have no competing interests.

\section{Authors' contributions}

All authors contributed to each part of this work equally and read and approved the final manuscript.

\section{Acknowledgements}

This study was partially supported by the Joint Funds of the National Natural Science Foundation of China (Grant No. U1204103). The authors would like to express their thanks to the reviewers for their helpful comments and suggestions

Received: 14 July 2014 Accepted: 12 September 2014 Published: 24 September 2014

\section{References}

1. Daubechies, I, Han, B, Ron, A, Shen, Z: Framelets: MRA-based constructions of wavelet frames. Appl. Comput. Harmon. Anal. 14(1), 1-46 (2003)

2. Dong, B, Shen, Z: Pseudo-splines, wavelets and framelets. Appl. Comput. Harmon. Anal. 22(1), 78-104 (2007)

3. Dong, B, Dyn, N, Hormann, K: Properties of dual pseudo-splines. Appl. Comput. Harmon. Anal. 29(1), 104-110 (2010)

4. Shen, Y, Li, S, Mo, Q: Complex wavelets and framelets from pseudo splines. J. Fourier Anal. Appl. 16(6), 885-900 (2010)

5. Cai, J-F, Shen, Z: Framelet based deconvolution. J. Comput. Math. 28(3), 289-308 (2010)

6. Cai, J, Chan, R, Shen, L, Shen, Z: Tight frame based method for high-resolution image reconstruction. In: Wavelet Methods in Mathematical Analysis and Engineering. Ser. Contemp. Appl. Math. CAM, vol. 14, pp. 1-36. Higher Ed. Press, Beijing (2010)

7. Daubechies, I: Ten Lectures on Wavelets. CBMS-NSF Regional Conference Series in Applied Mathematics, vol. 61. SIAM, Philadelphia (1992)

8. Deriaz, E, Perrier, V: Direct numerical simulation of turbulence using divergence-free wavelets. Multiscale Model Simul. 7(3), 1101-1129 (2008)

9. Chuang, Z-T, Liu, Y: Wavelets with differential relation. Acta Math. Sin. Engl. Ser. 27(5), 1011-1022 (2011)

10. Unser, M, Blu, T: Fractional splines and wavelets. SIAM Rev. 42(1), 43-67 (2000) (electronic)

11. Jia, RQ, Micchelli, CA: Using the refinement equations for the construction of pre-wavelets. II. Powers of two. In: Curves and Surfaces (Chamonix-Mont-Blanc, 1990), pp. 209-246. Academic Press, Boston (1991)

doi:10.1186/1029-242X-2014-359

Cite this article as: Zhuang and Yang: A class of generalized pseudo-splines. Journal of Inequalities and Applications 2014 2014:359. 